\title{
Erratum and addendum to: The $B V$-energy of maps into a manifold: relaxation and density results
}

\author{
Mariano Giaquinta And Domenico Mucci
}

In Step 3 of the proof of Theorem 2.14, in Section 4 of [1], there is a mistake. The same mistake also appears in [2, Section 7.5]. Using arguments from Step 3 and Step 4 of [1], here we correct such a mistake.

Letting $B_{j}=\bar{B}_{r}\left(x_{0}\right) \in \mathcal{F}_{m}^{\prime}$, we first deform the sliced current $\left\langle T, d_{x_{0}}, r\right\rangle$ to a Cartesian current $\widetilde{T}_{j}$ with support in $\partial B_{\delta}\left(x_{0}\right) \times B_{\mathcal{Y}}\left(y_{j}, \varepsilon_{m}\right)$, where $\delta<r$ and $B \mathcal{Y}\left(y_{j}, \varepsilon_{m}\right)$ is a small geodesic ball in $\mathcal{Y}$ centered at some point $y_{j} \in \mathcal{Y}$. We then regularize the boundary data $\widetilde{T}_{j}$ and apply a standard convolution and projection argument. For the reader's convenience, we present a quite complete outline of the proof.

Keeping Steps 1 and 2 as in Section 4 of [1], we proceed as follows.

Step 3: Projecting the boundary data. For any $\rho>0$, we set $Q_{\rho}^{n}:=[-\rho, \rho]^{n} \subset$ $\mathbb{R}^{n}$ and denote by $\Sigma_{\rho}^{i}$ the $i$-dimensional skeleton of $Q_{\rho}^{n}$, so that $\bigcup \Sigma_{\rho}^{n-1}=\partial Q_{\rho}^{n}$. Also, let $\|x\|:=\max \left\{\left|x_{1}\right|, \ldots,\left|x_{n}\right|\right\}$. In the sequel, we say that an $i$-dimensional current $S$ belongs to $\operatorname{cart}^{1,1}\left(\Sigma_{r}^{i} \times \mathcal{Y}\right)$ if for any $i$-face $F$ of $\Sigma_{r}^{i}$ its restriction $S\left\llcorner(F \times \mathcal{Y})\right.$ belongs to cart ${ }^{1,1}(F \times \mathcal{Y})$ and, for any $i$-faces $F_{1}$ and $F_{2}$ of $\Sigma_{r}^{i}$ that intersect on a common $(i-1)$-face $I$, we have

$$
\partial\left(S \llcorner ( F _ { 1 } \times \mathcal { Y } ) ) \left\llcornerI \times \mathcal{Y}=-\partial\left(S\left\llcorner\left(F_{2} \times \mathcal{Y}\right)\right)\llcorner I \times \mathcal{Y} .\right.\right.\right.
$$

In this case, moreover, we will denote by $\mathcal{E}_{1,1}\left(S, \Sigma_{r}^{i}\right)$ the sum of the $B V$-energies of the restrictions $S\left\llcorner(F \times \mathcal{Y})\right.$ of $S$ to all the $i$-faces $F$ of $\Sigma_{r}^{i}$. We also recall that $\mathcal{Y} \subset \mathbb{R}^{N}$, and denote by

$$
B \mathcal{Y}(y, \varepsilon):=\bar{B}^{N}(y, \varepsilon) \cap \mathcal{Y}
$$

the intersection of $\mathcal{Y}$ with the closed $N$-ball of radius $\varepsilon$ centered at $y$. If $y \in \mathcal{Y}$ and $0<\varepsilon<\varepsilon_{0}$, we let $\Psi_{(y, \varepsilon)}: \mathbb{R}^{N} \rightarrow \bar{B}_{\mathcal{Y}}(y, \varepsilon)$ be the retraction map given by $\Psi_{(y, \varepsilon)}(z):=\Pi_{\varepsilon} \circ \xi_{(y, \varepsilon)}$, where

$$
\xi_{(y, \varepsilon)}(z):=\left\{\begin{array}{llll}
z & \text { if } & z \in \bar{B}^{N}(y, \varepsilon) \\
\varepsilon \frac{z-y}{|z-y|} & \text { if } & z \in \mathbb{R}^{N} \backslash \bar{B}^{N}(y, \varepsilon)
\end{array}\right.
$$


and $\Pi_{\varepsilon}: \mathcal{Y}_{\varepsilon} \rightarrow \mathcal{Y}$ is the projection map given by Remark 1.9 in [1], so that $\Psi_{(y, \varepsilon)}$ is a Lipschitz continuous function with $\operatorname{Lip} \Psi_{(y, \varepsilon)}=\operatorname{Lip} \Pi_{\varepsilon} \rightarrow 1^{+}$as $\varepsilon \rightarrow 0^{+}$.

Let $B_{j}=\bar{B}_{r}\left(x_{0}\right) \in \mathcal{F}_{m}^{\prime}$. By means of a deformation and slicing argument, we may and do define a bilipschitz homeomorphism $\psi_{j}: \bar{B}_{r}\left(x_{0}\right) \rightarrow Q_{r}^{n}$ such that $\left\|D \psi_{j}\right\|_{\infty} \leq K,\left\|D \psi_{j}^{-1}\right\|_{\infty} \leq K$ for some absolute constant $K>0$, only depending on $n$, and

$$
\psi_{j}\left(\bar{B}_{\rho}\left(x_{0}\right)\right)=Q_{\rho}^{n} \quad \forall \rho \in(r / 2, r) .
$$

Setting

$$
T_{j}:=\left(\psi_{j} \bowtie I d_{\mathbb{R}^{N}}\right)_{\#}\left(T\left\llcorner\bar{B}_{r}\left(x_{0}\right) \times \mathcal{Y}\right)\right.
$$

we clearly have

$$
\partial T_{j}=\left(\psi_{j} \bowtie I d_{\mathbb{R}^{N}}\right)_{\#}\left\langle T, d_{x_{0}}, r\right\rangle \in \operatorname{cart}^{1,1}\left(\partial Q_{r}^{n} \times \mathcal{Y}\right) .
$$

Moreover, denoting by $T_{j}\left\llcorner\left(\Sigma_{r}^{i} \times \mathcal{Y}\right)\right.$ the $i$-dimensional slice of $T_{j}$ on $\Sigma_{r}^{i} \times \mathcal{Y}$, we also may and do define $\psi_{j}$ in such a way that $T_{j}\left\llcorner\left(\Sigma_{r}^{i} \times \mathcal{Y}\right)\right.$ is an $i$-dimensional current in $\operatorname{cart}^{1,1}\left(\Sigma_{r}^{i} \times \mathcal{Y}\right)$ satisfying the energy estimate

$$
\mathcal{E}_{1,1}\left(T_{j}, \Sigma_{r}^{i}\right) \leq C \cdot \frac{1}{r} \cdot \mathcal{E}_{1,1}\left(T_{j}, \Sigma_{r}^{i+1}\right) \quad \forall i=1, \ldots, n-2,
$$

where $C>0$ is an absolute constant, not depending on $T_{j}$, and $\mathcal{E}_{1,1}\left(T_{j}, \Sigma_{r}^{i}\right)$ denotes the $B V$-energy of the $i$-current $T_{j}\left\llcorner\left(\Sigma_{r}^{i} \times \mathcal{Y}\right)\right.$. With this notation, we have $T_{j}\left\llcorner\left(\Sigma_{r}^{n-1} \times \mathcal{Y}\right)=\partial T_{j}\right.$. Since by the construction

$$
\mathcal{E}_{1,1}\left(\left\langle T, d_{x_{0}}, r\right\rangle, \partial B_{r}\left(x_{0}\right) \times \mathcal{Y}\right) \leq \frac{2}{r} \mathcal{E}_{1,1}\left(T, \bar{B}_{2 r}\left(x_{0}\right) \times \mathcal{Y}\right)
$$

and

$$
\frac{1}{(2 r)^{n-1}} \mathcal{E}_{1,1}\left(T, \bar{B}_{2 r}\left(x_{0}\right) \times \mathcal{Y}\right) \leq \frac{1}{m},
$$

we infer that on one hand

$$
\mathcal{E}_{1,1}\left(T_{j}, \Sigma_{r}^{i}\right) \leq \widetilde{C} r^{i-n} \mathcal{E}_{1,1}\left(T, \bar{B}_{2 r}\left(x_{0}\right) \times \mathcal{Y}\right) \quad \forall i=1, \ldots, n-1
$$

and on the other hand

$$
\frac{1}{r^{i-1}} \mathcal{E}_{1,1}\left(T_{j}, \Sigma_{r}^{i}\right) \leq \widetilde{C} \frac{1}{m} \quad \forall i=1, \ldots, n,
$$

where $\widetilde{C}>0$ is an absolute constant. 
Remark 1.1. Let $u_{j}:=u_{T} \circ \psi_{j}^{-1}$ denote the $B V$-function corresponding to $T_{j}$, and $u_{j \mid \Sigma_{r}^{i}}$ the restriction of $u_{j}$ to $\Sigma_{r}^{i}$. The inequality (1.6), with $i=1$, yields on one hand that the concentration part of the 1-current $T_{j}\left\llcorner\Sigma_{r}^{1} \times \mathcal{Y}\right.$ is zero, and on the other hand that

$$
\left|D\left(u_{j \mid \Sigma_{r}^{1}}\right)\right|\left(\Sigma_{r}^{1}\right) \leq \widetilde{C} \frac{1}{m} .
$$

Therefore, setting $\varepsilon_{m}:=1 / \sqrt{m}$, for $m \in \mathbb{N}$ sufficiently large the image $u_{j}\left(\Sigma_{r}^{1}\right)$ is contained in a small geodesic ball $B \mathcal{Y}\left(y_{j}, \varepsilon_{m} / 2\right)$ centered at some given point $y_{j} \in \mathcal{Y}$. As a consequence, we obtain that

$$
\operatorname{spt}\left(T_{j}\left\llcorner\left(\Sigma_{r}^{1} \times \mathcal{Y}\right)\right) \subset \Sigma_{r}^{1} \times \bar{B} \mathcal{Y}\left(y_{j}, \varepsilon_{m} / 2\right) .\right.
$$

Let $q \in \mathbb{N}^{+}$. In the case of dimension $n \geq 3$, following an argument by Bethuel, if $S_{h}$ is one of the $(n-1)$-faces of $\Sigma_{r}^{n-1}$, where $h=1, \ldots, 2 n$, we may and do define a partition of $S_{h}$ into $(q+1)^{n-1}$ small $(n-1)$-dimensional "cubes" $C_{l, h}$ in such a way that the following facts hold:

i) If $\left[C_{l, h}\right]_{i}$ denotes the $i$-dimensional skeleton of the boundary of $C_{l, h}$, the restriction of $T_{j}$ to $\left[C_{l, h}\right]_{i} \times \mathcal{Y}$ is an $i$-dimensional current in $\operatorname{cart}^{1,1}\left(\left[C_{l, h}\right]_{i} \times \mathcal{Y}\right)$ for every $i=1, \ldots, n-2$.

ii) If $n=3$, we have

$$
\sum_{l=1}^{(q+1)^{2}} \mathcal{E}_{1,1}\left(T_{j}, \partial C_{l, h}\right) \leq K\left(\mathcal{E}_{1,1}\left(T_{j}, \partial S_{h}\right)+\frac{q}{r} \mathcal{E}_{1,1}\left(T_{j}, S_{h}\right)\right),
$$

where $K>0$ is an absolute constant.

iii) If $n \geq 4$, and $\left[S_{h}\right]_{i}$ denotes the $i$-dimensional skeleton of $S_{h}$, for every $i=$ $1, \ldots, n-2$ we have

$$
\sum_{l=1}^{(q+1)^{n-1}} \mathcal{E}_{1,1}\left(T_{j},\left[C_{l, h}\right]_{i}\right) \leq K \cdot \sum_{t=i}^{n-1}\left(\frac{q}{r}\right)^{t-i} \cdot \mathcal{E}_{1,1}\left(T_{j},\left[S_{h}\right]_{t}\right),
$$

where $K>0$ is an absolute constant.

iv) All the $C_{l, h}$ 's are bilipschitz homeomorphic to the $(n-1)$-cube $[-r / q, r / q]^{n-1}$ by linear maps $f_{l, h}$ such that $\left\|D f_{l, h}\right\|_{\infty} \leq K,\left\|D f_{l, h}^{-1}\right\|_{\infty} \leq K$.

Remark 1.2. By (1.6) and (1.7), or (1.8), we infer that

$$
\sum_{l=1}^{(q+1)^{n-1}} \mathcal{E}_{1,1}\left(T_{j},\left[C_{l, h}\right]_{1}\right) \leq \widehat{C} \frac{q^{n-2}}{m},
$$

where $\widehat{C}>0$ is an absolute constant. Moreover, the image $u_{j}\left(\Sigma_{r}^{1}\right)$ is contained in $B \mathcal{Y}\left(y_{j}, \varepsilon_{m} / 2\right)$. Therefore, in the sequel we will take

$$
q:=\text { integer part of }\left((2 \widehat{C})^{-1} \cdot \varepsilon_{m} \cdot m\right)^{1 /(n-2)} .
$$


Arguing as in Remark 1.1, we then infer that the image of $\left[C_{l, h}\right]_{1}$ by $u_{j}$ is contained in the geodesic ball $B \mathcal{Y}\left(y_{j}, \varepsilon_{m}\right)$ and, moreover, that

$$
\operatorname{spt}\left(T_{j}\left\llcorner\left(\left[C_{l, h}\right]_{1} \times \mathcal{Y}\right)\right) \subset\left[C_{l, h}\right]_{1} \times \bar{B}_{\mathcal{Y}}\left(y_{j}, \varepsilon_{m}\right)\right.
$$

for every $l$ and $h$.

Let $\delta:=r\left(1-q^{-1}\right)$ and define $\Phi_{q}: Q_{r}^{n} \rightarrow Q_{\delta}^{n}$ by $\Phi_{q}(x):=\left(1-q^{-1}\right) x$ and $\pi_{(r, \delta)}: Q_{r}^{n} \backslash Q_{\delta}^{n} \rightarrow \partial Q_{r}^{n}$ by $\pi_{(r, \delta)}(x):=r x /\|x\|$. Setting

$$
\mathcal{M}_{(r, \delta)}:=\pi_{(r, \delta)}^{-1}\left(\bigcup_{h=1}^{2 n} \bigcup_{l=1}^{(q+1)^{n-1}} \partial C_{l, h}\right)
$$

it turns out that the $(n-1)$-skeleton

$$
\mathcal{N}_{(r, \delta)}:=\mathcal{M}_{(r, \delta)} \cup \partial Q_{r}^{n} \cup \partial Q_{\delta}^{n}
$$

is the union of the boundaries of $n$-dimensional "cubes" $Q_{l, h}$, satisfying $C_{l, h} \subset$ $\partial Q_{l, h}$ for every $l$ and $h$, that partition $Q_{r}^{n} \backslash Q_{\delta}^{n}$. Moreover, each $Q_{l, h}$ is bilipschitz homeomorphic to the $n$-cube $[-r / q, r / q]^{n}$ by linear maps $\widetilde{f}_{l, h}$ such that $\left\|D \widetilde{f}_{l, h}\right\|_{\infty} \leq K,\left\|D \tilde{f}_{l, h}^{-1}\right\|_{\infty} \leq K$, where $K>0$ is an absolute constant. Finally, set

$$
\widetilde{\Sigma}_{r}^{i}:=\left(\bigcup_{h=1}^{2 n} \bigcup_{l=1}^{(q+1)^{n-1}}\left[C_{l, h}\right]_{i}\right)
$$

and denote by $\mathcal{N}_{(r, \delta)}^{i}$ the $i$-dimensional skeleton of $\mathcal{N}_{(r, \delta)}$, so that

$$
\mathcal{N}_{(r, \delta)}^{i}=\widetilde{\Sigma}_{r}^{i} \cup \Phi_{q}\left(\widetilde{\Sigma}_{r}^{i}\right) \cup \pi_{(r, \delta)}^{-1}\left(\widetilde{\Sigma}_{r}^{i-1}\right) \quad \forall i=2, \ldots, n-1
$$

We now define an $n$-current $\widehat{T}_{j}$ in $\operatorname{cart}^{1,1}\left(\operatorname{int}\left(Q_{r}^{n} \backslash Q_{\delta}^{n}\right) \times \mathcal{Y}\right)$ and an $(n-1)$ current $\widetilde{T}_{j} \in \operatorname{cart}^{1,1}\left(\partial Q_{\delta}^{n} \times \mathcal{Y}\right)$ such that the following properties hold:

(a) $\widehat{T}_{j}$ has small $B V$-energy;

(b) $\widetilde{T}_{j}$ is supported in $\partial Q_{\delta}^{n} \times \bar{B} \mathcal{Y}\left(y_{j}, \varepsilon_{m}\right)$ and its $B V$-energy is comparable to the $B V$-energy of $\partial T_{j}$

(c) the boundary of $\widehat{T}_{j}$ agrees with $\partial T_{j}$ on $\partial Q_{r}^{n} \times \mathcal{Y}$;

(d) the boundary of $\widehat{T}_{j}$ agrees with $-\widetilde{T}_{j}$ on $\partial Q_{\delta}^{n} \times \mathcal{Y}$.

To this purpose we first define a 2-current $S_{j}^{(2)}$ on $\mathcal{N}_{(r, \delta)}^{2} \times \mathcal{Y}$ by setting

$$
S_{j}^{(2)}:=\left\{\begin{array}{lll}
T_{j}\left\llcorner\left(\widetilde{\Sigma}_{r}^{2} \times \mathcal{Y}\right)\right. & \text { on } & \widetilde{\Sigma}_{r}^{2} \times \mathcal{Y} \\
\left(\Phi_{q} \bowtie \Psi_{\left(y_{j}, \varepsilon_{m}\right)}\right)_{\#} T_{j}\left\llcorner\left(\widetilde{\Sigma}_{r}^{2} \times \mathcal{Y}\right)\right. & \text { on } & \Phi_{q}\left(\widetilde{\Sigma}_{r}^{2}\right) \times \mathcal{Y}
\end{array}\right.
$$


whereas on $\pi_{(r, \delta)}^{-1}\left(\widetilde{\Sigma}_{r}^{1}\right) \times \mathcal{Y}$ we set

$$
S_{j}^{(2)}:=\sum_{h=1}^{2 n} \sum_{l=1}^{(q+1)^{n-1}} H_{\#}\left(\llbracket 0,1 \rrbracket \times\left(T_{j}\left\llcorner\left(\left[C_{l, h}\right]_{1} \times \mathcal{Y}\right)\right)\right)\right.
$$

where $H$ is the affine homotopy map

$$
H(t, x, y):=\left(t \delta \frac{x}{\|x\|}+(1-t) x, y\right), \quad t \in[0,1], x \in\left[C_{l, h}\right]_{1}, y \in \mathcal{Y}
$$

By (1.10) and (1.11) we infer that $S_{j}^{(2)}$ is well-defined in $\mathcal{N}_{(r, \delta)}^{2} \times \mathcal{Y}$ in such a way (1.1) holds, with $S=S_{j}^{(2)}$, for every 2-faces $F_{1}$ and $F_{2}$ of $\mathcal{N}_{(r, \delta)}^{2}$ that intersect on a common 1-face $I$.

The case $n=3$. We then define $\widehat{T}_{j}$ on each $Q_{l, h}$ by

$$
\widehat{T}_{j}\left\llcorner\left(Q_{l, h} \times \mathcal{Y}\right):=\widehat{H}_{\#}\left(\llbracket 0,1 \rrbracket \times\left(S_{j}^{(2)}\left\llcorner\left(\partial Q_{l, h} \times \mathcal{Y}\right)\right)\right),\right.\right.
$$

where $\widehat{H}(t, x, y):=\left(t c_{l, h}+(1-t) x, y\right)$ and $c_{l, h}$ is the barycenter of $Q_{l, h}$. On account of (1.1), with $S=S_{j}^{(2)}$, we infer that the current $\widehat{T}_{j}\left\llcorner\operatorname{int}\left(Q_{l, h}\right) \times\right.$ $\mathcal{Y}$ actually belongs to cart ${ }^{1,1}$ for every $h$ and $l$. In fact, the boundary of $\widehat{T}_{j}$ is computed on 2 -forms in $\mathcal{Z}^{2,1}$, whence it cannot see the "singular" set $\left\{c_{l, h}\right\} \times \mathcal{Y}$ of $\widehat{T}_{j}$. Moreover, it is readily checked that $\widehat{T}_{j}$ satisfies the energy estimate

$$
\mathcal{E}_{1,1}\left(\widehat{T}_{j}, \operatorname{int}\left(Q_{l, h}\right) \times \mathcal{Y}\right) \leq C \frac{r}{q} \mathcal{E}_{1,1}\left(S_{j}^{(2)}, \partial Q_{l, h}\right)
$$

whereas by the definition of $S_{j}^{(2)}$ we obtain

$$
\mathcal{E}_{1,1}\left(S_{j}^{(2)}, \partial Q_{l, h}\right) \leq C\left(\mathcal{E}_{1,1}\left(T_{j}, C_{l, h}\right)+\frac{r}{q} \mathcal{E}_{1,1}\left(T_{j}, \partial C_{l, h}\right)\right) .
$$

Therefore, by (1.7), and by summing on $l$ and $h$, we estimate

$$
\mathcal{E}_{1,1}\left(\widehat{T}_{j},\left(Q_{r}^{3} \backslash Q_{\delta}^{3}\right) \times \mathcal{Y}\right) \leq C\left(\frac{r}{q} \mathcal{E}_{1,1}\left(T_{j}, \Sigma_{r}^{2}\right)+\left(\frac{r}{q}\right)^{2} \mathcal{E}_{1,1}\left(T_{j}, \Sigma_{r}^{1}\right)\right) .
$$

In conclusion, for $m$ large, and $n=3$, by (1.9) and (1.5) we obtain the energy estimate

$$
\mathcal{E}_{1,1}\left(\widehat{T}_{j},\left(Q_{r}^{n} \backslash Q_{\delta}^{n}\right) \times \mathcal{Y}\right) \leq C\left(\varepsilon_{m} \cdot m\right)^{1 /(2-n)} \mathcal{E}_{1,1}\left(T, \bar{B}_{2 r}\left(x_{0}\right) \times \mathcal{Y}\right)
$$

where, we recall, $\left(\varepsilon_{m} \cdot m\right)^{1 /(2-n)} \rightarrow 0$ as $m \rightarrow+\infty$, since $\varepsilon_{m} \cdot m=\sqrt{m}$. 
The case $n \geq 4$. We define an $i$-current $S_{j}^{(i)}$ on $\mathcal{N}_{(r, \delta)}^{i} \times \mathcal{Y}$ arguing by iteration on the dimension $i=3, \ldots, n-1$. More precisely, if $F$ is any $i$-face of $\left[Q_{l, h}\right]_{i}$, we distinguish two cases. If $F$ is contained in $\partial Q_{r}^{n}$ we set

$$
S_{j}^{(i)}\left\llcorner(F \times \mathcal{Y}):=T_{j}\llcorner(F \times \mathcal{Y}) .\right.
$$

Otherwise, we define $S_{j}^{(i)}$ on $F \times \mathcal{Y}$ by means of a "radial" extension of the boundary datum $S_{j}^{(i-1)}\llcorner(\partial F \times \mathcal{Y})$ similar to the one in (1.12), so that

$$
\mathcal{E}_{1,1}\left(S_{j}^{(i)}, F\right) \leq C \frac{r}{q} \mathcal{E}_{1,1}\left(S_{j}^{(i-1)}, \partial F\right) .
$$

Notice that for every $(i-1)$-faces $F_{1}$ and $F_{2}$ of $\mathcal{N}_{(r, \delta)}^{i-1}$ that intersect on a common $(i-2)$-face $I$, we again have that (1.1) holds true, with $S=S_{j}^{(i-1)}$. We finally define $\widehat{T}_{j}$ on each $Q_{l, h}$ by (1.12), with $S_{j}^{(n-1)}$ instead of $S_{j}^{(2)}$. By the construction, and for (1.8), we readily infer that

$$
\mathcal{E}_{1,1}\left(\widehat{T}_{j},\left(Q_{r}^{n} \backslash Q_{\delta}^{n}\right) \times \mathcal{Y}\right) \leq C \sum_{i=1}^{n-1}\left(\frac{r}{q}\right)^{n-i} \mathcal{E}_{1,1}\left(T_{j}, \Sigma_{r}^{i}\right),
$$

so that by (1.9) and (1.5) we obtain again (1.13), for $m$ large. Now, for any $n \geq 3$ the current $\widehat{T}_{j}$ this way constructed belongs to $\operatorname{cart}{ }^{1,1}\left(\operatorname{int}\left(Q_{r}^{n} \backslash Q_{\delta}^{n}\right), \mathcal{Y}\right)$. In fact, the boundary of $\widehat{T}_{j}$ is computed on $(n-1)$-forms in $\mathcal{Z}^{n-1,1}$, hence it cannot see a "singular" set that lives on $\Sigma \times \mathcal{Y}$ for some $(n-3)$-dimensional skeleton $\Sigma$. Moreover, the above properties (a)-(d) follow from the construction, as required.

In conclusion, setting

$$
S_{j}:=\left(\psi_{j}^{-1} \bowtie I d_{\mathbb{R}^{N}}\right)_{\#}\left(\widehat{T}_{j}\left\llcorner\operatorname{int}\left(Q_{r}^{n} \backslash Q_{\delta}^{n}\right) \times \mathcal{Y}\right),\right.
$$

on account of (1.2) we infer that $S_{j}$ belongs to $\operatorname{cart}^{1,1}\left(\left(B_{r}\left(x_{0}\right) \backslash \bar{B}_{\delta}\left(x_{0}\right)\right) \times \mathcal{Y}\right)$, and by (1.13) it satisfies the energy estimate

$$
\mathcal{E}_{1,1}\left(S_{j},\left(B_{r}\left(x_{0}\right) \backslash \bar{B}_{\delta}\left(x_{0}\right)\right) \times \mathcal{Y}\right) \leq C\left(\varepsilon_{m} \cdot m\right)^{1 /(2-n)} \mathcal{E}_{1,1}\left(T, \bar{B}_{2 r}\left(x_{0}\right) \times \mathcal{Y}\right) .
$$

Finally, by the properties (c) (d) we infer that $S_{j}$ satisfies the boundary conditions

$$
\partial S_{j}\left\llcorner\left(\partial B_{r}\left(x_{0}\right) \times \mathcal{Y}\right)=\left\langle T, d_{x_{0}}, r\right\rangle\right.
$$

and

$$
\partial S_{j}\left\llcorner\left(\partial B_{\delta}\left(x_{0}\right) \times \mathcal{Y}\right)=-\widetilde{T}_{j},\right.
$$

where $\widetilde{T}_{j} \in \operatorname{cart}^{1,1}\left(\partial B_{\delta}\left(x_{0}\right) \times \mathcal{Y}\right)$ has support

$$
\operatorname{spt} \widetilde{T}_{j} \subset \partial B_{\delta}\left(x_{0}\right) \times B \mathcal{Y}\left(y_{j}, \varepsilon_{m}\right)
$$


and $B V$-energy

$$
\mathcal{E}_{1,1}\left(\widetilde{T}_{j}, \partial B_{\delta}\left(x_{0}\right) \times \mathcal{Y}\right) \leq C \cdot \mathcal{E}_{1,1}\left(\left\langle T, d_{x_{0}}, r\right\rangle, \partial B_{r}\left(x_{0}\right) \times \mathcal{Y}\right) .
$$

In the case of dimension $n=2$ we simply take $\delta=r$ and $\widetilde{T}_{j}:=\left\langle T, d_{x_{0}}, r\right\rangle$. The energy bounds (1.3) and (1.4) yield that (1.15) holds true, see Remark 1.1.

Step 4: Approximation on the balls of $\mathcal{F}_{m}^{\prime}$. Set $\widehat{B}_{j}:=B_{\delta}\left(x_{0}\right)$ and let $v_{j} \in$ $B V\left(\partial \widehat{B}_{j}, \mathcal{Y}\right)$ denote the $B V$-function corresponding to $\widetilde{T}_{j}$. Using the argument in Step 3 of [1, Section 4], due to the inductive hypothesis, we find a sequence of smooth maps $\left\{v_{h}^{(j)}\right\} \subset W^{1,1}\left(\partial \widehat{B}_{j}, \mathcal{Y}\right)$ such that $\left\|v_{h}^{(j)}-v_{j}\right\|_{L^{1}\left(\partial \widehat{B}_{j}\right)} \rightarrow 0$,

$$
G_{v_{h}^{(j)}} \rightarrow \widetilde{T}_{j} \quad \text { weakly in } \quad \mathcal{Z}_{n-1,1}\left(\partial \widehat{B}_{j} \times \mathcal{Y}\right)
$$

as $h \rightarrow \infty$ and

$$
\int_{\partial \widehat{B}_{j}}\left|D_{\tau} v_{h}^{(j)}\right| d \mathcal{H}^{n-1} \leq \mathcal{E}_{1,1}\left(\widetilde{T}_{j}, \partial \widehat{B}_{j} \times \mathcal{Y}\right) \cdot\left(1+2^{-h}\right)
$$

for every $h$. By property (1.15), we may and do assume that the approximating sequence satisfies

$$
v_{h}^{(j)}\left(\partial \widehat{B}_{j}\right) \subset B \mathcal{Y}\left(y_{j}, \varepsilon_{m}\right)
$$

for every $h$. Taking $k$ sufficiently large, and using the argument by Gagliardo, we then define a map $W_{k}^{(j)} \in W^{1,1}\left(A_{\rho_{k}}^{\delta}, \mathbb{R}^{N}\right)$, where $0<\rho_{k}<\delta$ and $A_{\rho}^{R}=A_{\rho}^{R}\left(x_{0}\right)$ denotes the annulus

$$
A_{\rho}^{R}:=\bar{B}_{R}\left(x_{0}\right) \backslash B_{\rho}\left(x_{0}\right), \quad 0<\rho<R,
$$

in such a way that $W_{k \mid \partial B_{\delta}\left(x_{0}\right)}^{(j)}=v_{j \mid \partial B_{\delta}\left(x_{0}\right)}$ in the sense of traces,

$$
W_{k}^{(j)}\left(x_{0}+\rho_{k} \frac{x-x_{0}}{\left|x-x_{0}\right|}\right)=v_{k}^{(j)}\left(x_{0}+\delta \frac{x-x_{0}}{\left|x-x_{0}\right|}\right)
$$

and the energy $\int_{A_{\rho_{k}}^{\delta}}\left|D W_{k}^{(j)}\right| d x$ is arbitrarily small, if $\rho_{k} \nearrow \delta$ sufficiently rapidly. Condition (1.16) yields

$$
\operatorname{dist}\left(W_{k}^{(j)}(x), \mathcal{Y}\right)<\varepsilon_{0} \quad \text { for } \mathcal{L}^{n} \text {-a.e. } x \in A_{\rho_{k}}^{\delta}
$$

for $m$ large enough, hence we may and do define $w_{k}^{(j)}:=\Pi_{\varepsilon_{0}} \circ W_{k}^{(j)}$ on $A_{\rho_{k}}^{\delta}$, where $\Pi_{\varepsilon_{0}}$ is the Lipschitz projection onto $\mathcal{Y}$ given by Remark 1.9 in [1], so that $w_{k}^{(j)}\left(A_{\rho_{k}}^{\delta}\right) \subset B \mathcal{Y}\left(y_{j}, \varepsilon_{m}\right)$. 
We now extend $w_{k}^{(j)}$ to the whole ball $\widehat{B}_{j}$ by the map $\widetilde{w}_{k}^{(j)}: \bar{B}_{\rho_{k}}\left(x_{0}\right) \rightarrow$ $B \mathcal{Y}\left(y_{j}, \varepsilon_{m}\right)$ given by

$$
\widetilde{w}_{k}^{(j)}(x):=\left\{\begin{array}{lll}
w_{k}^{(j)} \circ \psi_{(\delta, \sigma)}(x) & \text { if } \quad x \in A_{\delta-2 \sigma}^{\delta-\sigma} \\
\Psi_{\left(y_{j}, \varepsilon_{m}\right)} \circ u \circ \phi_{(\delta, \sigma, r)}(x) & \text { if } \quad x \in B_{\delta-2 \sigma}\left(x_{0}\right),
\end{array}\right.
$$

where $u=u_{T}, \sigma:=\delta-\rho_{k}, \psi_{(\delta, \sigma)}: A_{\delta-2 \sigma}^{\delta-\sigma} \rightarrow A_{\delta-\sigma}^{\delta}$ is the reflection map

$$
\psi_{(\delta, \sigma)}(x):=\left(-\left|x-x_{0}\right|+2(\delta-\sigma)\right) \frac{x-x_{0}}{\left|x-x_{0}\right|}
$$

and $\phi_{(\delta, \sigma, r)}: B_{\delta-2 \sigma}\left(x_{0}\right) \rightarrow B_{r}\left(x_{0}\right)$ is the homothetic map

$$
\phi_{(\delta, \sigma)}(x):=x_{0}+\frac{r}{\delta-2 \sigma}\left(x-x_{0}\right) .
$$

Set now $\rho:=\rho_{k}=\delta-\sigma$. Since the image of $B_{\rho}\left(x_{0}\right)$ by $\widetilde{w}_{k}^{(j)}$ is contained in the geodesic ball $B \mathcal{Y}\left(y_{j}, \varepsilon_{m}\right)$, by means of a convolution argument we can approximate $\widetilde{w}_{k}^{(j)}$ on $B_{\rho}\left(x_{0}\right)$ by a smooth sequence $v_{\varepsilon}^{(j)}: B_{\rho}\left(x_{0}\right) \rightarrow \bar{B}^{N}\left(y_{j}, \varepsilon_{m}\right)$ that converges in the $L^{1}$-sense to $\widetilde{w}_{k \mid B_{\rho}\left(x_{0}\right)}^{(j)}$ and with total variation converging to the total variation $\left|D \widetilde{w}_{k}^{(j)}\right|\left(B_{\rho}\left(x_{0}\right)\right)$. We finally set $w_{\varepsilon}^{(j)}:=\Pi_{\varepsilon_{m}} \circ v_{\varepsilon}^{(j)}: B_{\rho}\left(x_{0}\right) \rightarrow$ $B \mathcal{Y}\left(y_{j}, \varepsilon_{m}\right)$, so that clearly $w_{\varepsilon}^{(j)} \rightarrow \widetilde{w}_{k}^{(j)}$ weakly in $B V\left(B_{\rho}\left(x_{0}\right), \mathbb{R}^{N}\right)$, whereas

$$
\left|D w_{\varepsilon}^{(j)}\right|\left(B_{\rho}\left(x_{0}\right)\right) \leq\left(\operatorname{Lip} \Pi_{\varepsilon_{m}}\right) \cdot\left|D v_{\varepsilon}^{(j)}\right|\left(B_{\rho}\left(x_{0}\right)\right) .
$$

Therefore, the energy of $\widetilde{w}_{k}^{(j)}$ being small on $A_{\delta-2 \sigma}^{\delta-\sigma}$, we may and do assume that

$$
\limsup _{\varepsilon \rightarrow 0}\left|D w_{\varepsilon}^{(j)}\right|\left(B_{\rho}\left(x_{0}\right)\right) \leq\left(\operatorname{Lip} \Pi_{\varepsilon_{m}}\right)^{2} \cdot|D u|\left(B_{r}\left(x_{0}\right)\right)+\frac{2^{-j}}{k} .
$$

Moreover, by suitably defining the convolution kernel, we may and do assume that the traces are equal, so that $w_{\varepsilon \mid \partial B_{\rho}\left(x_{0}\right)}^{(j)}=v_{\varepsilon \mid \partial B_{\rho}\left(x_{0}\right)}^{(j)}=\widetilde{w}_{k \mid \partial B_{\rho}\left(x_{0}\right)}^{(j)}$. Most importantly, by the construction we may and do assume that the boundaries of the graphs agree on $\partial B_{\rho}\left(x_{0}\right)$, so that

$$
\partial G_{w_{\varepsilon}^{(j)}}\left\llcorner\partial B_{\rho}\left(x_{0}\right) \times \mathcal{Y}=\partial G_{v_{\varepsilon}^{(j)}}\left\llcorner\partial B_{\rho}\left(x_{0}\right) \times \mathcal{Y}=\partial G_{\widetilde{w}_{k}^{(j)}}\left\llcorner\partial B_{\rho}\left(x_{0}\right) \times \mathcal{Y} .\right.\right.\right.
$$

We then define $u_{k}^{(j)}: \bar{B}_{\delta}\left(x_{0}\right) \rightarrow \mathcal{Y}$ by

$$
u_{k}^{(j)}(x):=\left\{\begin{array}{lll}
w_{k}^{(j)}(x) & \text { if } & x \in A_{\rho_{k}}^{\delta} \\
w_{\varepsilon_{k}}^{(j)}(x) & \text { if } & x \in B_{\rho_{k}}\left(x_{0}\right)
\end{array}\right.
$$


where $\rho=\rho_{k}$ and $\varepsilon_{k} \searrow 0$ along a sequence. Moreover, for every $m$ and $k$ we let $T_{k}^{(m)} \in \mathcal{D}_{n, 1}\left(B^{n} \times \mathcal{Y}\right)$ be given by

$$
T_{k}^{(m)}:=T\left\llcorner\left(B^{n} \backslash \Omega_{m}\right) \times \mathcal{Y}+\sum_{j=1}^{\infty}\left(S_{j}+G_{u_{k}^{(j)}}\left\llcorner\operatorname{int}\left(\widehat{B}_{j}\right) \times \mathcal{Y}\right),\right.\right.
$$

where $S_{j}$ is defined in Step 3, so that $T_{k}^{(m)} \in \operatorname{cart}^{1,1}\left(B^{n} \times \mathcal{Y}\right)$.

Step 5: Approximating maps on the whole domain. As in Step 5 of [1, Section 4], a part from the fact that the Cantor part of $D u_{m}$ is not zero on the annuli $B_{r}\left(x_{0}\right) \backslash$ $B_{\delta}\left(x_{0}\right)$. However, due to the energy estimate (1.13), by summing on $j$, we may and do assume that for $m$ large enough

$$
\left|D^{C} u_{m}\right|\left(B^{n}\right) \leq \frac{1}{2}\left|D^{C} u_{T}\right|\left(B^{n}\right) .
$$

Step 6: Approximating currents. Set $T_{m}:=T_{k_{m}}^{(m)}$, where the sequence $k_{m} \rightarrow \infty$ is defined as in Step 5. We show that the flat distance of $T_{m}$ from $T$ is small. Recall that the flat norm $T \mapsto \mathbf{F}(T)$ is given by

$$
\mathbf{F}(T):=\sup \left\{T(\phi) \mid \phi \in \mathcal{Z}^{n-1,1}\left(B^{n} \times \mathcal{Y}\right), \mathbf{F}(\phi) \leq 1\right\},
$$

where

$$
\mathbf{F}(\phi):=\max \left\{\sup _{z \in B^{n} \times \mathcal{Y}}\|\phi(z)\|, \sup _{z \in B^{n} \times \mathcal{Y}}\|d \phi(z)\|\right\} .
$$

In fact, by (1.21) we infer that

$$
\mathbf{F}\left(T_{k}^{(m)}-T\right) \leq \sum_{j=1}^{\infty} \mathbf{F}\left(\left(T_{k}^{(m)}-T\right)\left\llcorner\operatorname{int}\left(B_{j}\right) \times \mathcal{Y}\right)\right) .
$$

Moreover, condition

$$
\mu_{J c}\left(J_{c}(T) \backslash J_{m}\right)<\frac{1}{m}
$$

yields that the Jump-concentration part of the energy of $T$ and of $T_{k}^{(m)}$ is small on the union of the balls $B_{j}$. Therefore, using the $L^{1}$-convergence of $u_{m}$ to $u$, for every $\varepsilon \in(0,1)$, possibly passing to a subsequence, we have

$$
\mathbf{F}\left(T_{m}-T\right) \leq \varepsilon^{m} \quad \forall m .
$$

On account of (1.22), and using an iteration argument similar to the one used in [1, Section 5] to obtain Theorem 2.15 from Proposition 5.1, we find the approximating sequence $\left\{T_{k}\right\}$ such that $u_{k}:=u_{T_{k}}$ in $B V\left(B^{n}, \mathcal{Y}\right)$ has no Cantor part, $\left|D^{C} u_{k}\right|=$ 0 for every $k$. 


\section{References}

[1] M. Giaquinta and D. MUCCI, The BV-energy of maps into a manifold: relaxation and density results, Ann. Scuola Norm. Sup. Pisa Cl. Sci. (5) 5 (2006), 483-548.

[2] M. Giaquinta and D. MucCi, Maps into manifolds and currents: area and $W^{1,2}-, W^{1 / 2}$-, $B V$-energies, Edizioni della Normale, C.R.M. Series, Scuola Norm. Sup. Pisa, 2006.

Scuola Normale Superiore

Piazza dei Cavalieri, 7

56100 Pisa, Italy

giaquinta@sns.it

Dipartimento di Matematica dell'Università di Parma Viale G. P. Usberti, 53/A

43100 Parma, Italy

domenico.mucci@unipr.it 